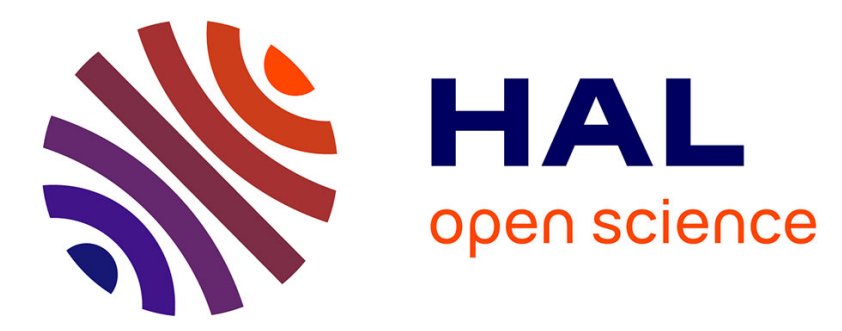

\title{
Characterizing ultrashort laser pulses by the rotational Doppler effect
}

E Szmygel, P Béjot, A Dubrouil, F Billard, B Lavorel, O Faucher, E Hertz

\section{To cite this version:}

E Szmygel, P Béjot, A Dubrouil, F Billard, B Lavorel, et al.. Characterizing ultrashort laser pulses by the rotational Doppler effect. Physical Review A, 2021, 104, 10.1103/physreva.104.013514 . hal03369988

\section{HAL Id: hal-03369988 \\ https://hal.science/hal-03369988}

Submitted on 7 Oct 2021

HAL is a multi-disciplinary open access archive for the deposit and dissemination of scientific research documents, whether they are published or not. The documents may come from teaching and research institutions in France or abroad, or from public or private research centers.
L'archive ouverte pluridisciplinaire HAL, est destinée au dépôt et à la diffusion de documents scientifiques de niveau recherche, publiés ou non, émanant des établissements d'enseignement et de recherche français ou étrangers, des laboratoires publics ou privés. 


\title{
Characterizing ultrashort laser pulses by the rotational Doppler effect
}

\author{
E. Szmygel $\odot,{ }^{1,2}$ P. Béjot, ${ }^{1}$ A. Dubrouil, ${ }^{2}$ F. Billard $\odot,{ }^{1}$ B. Lavorel,,${ }^{1}$ O. Faucher, ${ }^{1}$ and E. Hertz $\circledast^{1, *}$ \\ ${ }^{1}$ Laboratoire Interdisciplinaire Carnot de Bourgogne, UMR CNRS 6303, Université Bourgogne Franche-Comté, 21078 Dijon Cedex, France \\ ${ }^{2}$ Femto Easy, Batiment Gienah, Cité de la Photonique, 11 avenue de Canteranne, 33600 Pessac, France
}

(Received 9 April 2021; accepted 23 June 2021; published 14 July 2021)

\begin{abstract}
Technological advances in femtosecond laser sources call for the development of increasingly refined characterization tools implying to enrich the existing panel of operable nonlinear interactions. Toward that end, we have recently proposed a variant of SPIDER (spectral phase interferometry for direct electric field reconstruction) based on a nonstandard effect for producing the frequency shear, the so-called rotational Doppler effect. The method called DEER-SPIDER, for Doppler effect E-field replication, has the advantage of producing a spectral shearing at/near the fundamental wavelength, thus allowing operation in the ultraviolet spectral range. The present paper provides a deeper study of this approach. The method is tested under two different challenging conditions, and a thorough theoretical analysis is proposed. Possible improvements and an outlook are also discussed.
\end{abstract}

DOI: 10.1103/PhysRevA.104.013514

\section{INTRODUCTION}

The Doppler effect [1] is mostly known in its linear form, also called the "translational Doppler effect," by which the relative velocity between a wave source and an observer causes a frequency shift readily understood from the conservation of linear momentum and energy during the light-matter interaction. The translational Doppler effect is widely used for various applications, such as laser remote sensing, laser velocimetry, health care, satellite communication, and robotics [2-5]. In astronomy, the discovery of exoplanets by Doppler spectroscopy was awarded by the Nobel Prize in physics in 2019 [6]. Another lesser known manifestation of the Doppler effect is the "rotational Doppler effect," which has been the subject of a growing interest during the past decade. The frequency shift arises in this case from the emission or absorption of a circularly polarized light by a rotating body and is explained from the conservation of angular momentum and energy. The rotational Doppler effect manifests itself through various physical processes such as rotational scattering [7]. It has also been used in photoelectron spectroscopy for probing molecular orbitals anisotropy [8], in satellite-based GPS navigation systems [9], or to measure the angular speed of coherently spinning molecules [10-13]. We have recently reported [14] its relevance in a radically different field of applications, namely the ultrashort pulse characterization, by proposing a variant of the spectral phase interferometry for direct electric field reconstruction (SPIDER). The method is called Doppler effect E-field replication-SPIDER (DEERSPIDER). The SPIDER represents one of the most widespread self-referenced complete characterization methods with the frequency-resolved optical gating (FROG) $[15,16]$ and to a lesser extent the d-scan [17] or the self-referenced spectral

\footnotetext{
*edouard.hertz@u-bourgogne.fr
}

interferometry (SRSI) [18]. The SPIDER consists of measuring the spectral interferometry between a pair of identical but frequency-sheared replicas of the input pulse. In the original version of the SPIDER, the input pulse is split into two parts, one being used to produce a highly chirped pulse and the other one to generate a sequence of two time-delayed pulses. The two replica are then sum frequency mixed in a doubling crystal with the chirped pulse. Since each pulse overlaps with a different quasimonochromatic slice of the chirped pulse, the generated pulse has a slightly different central frequency (near twice the fundamental carrier frequency). The frequency shear $\Omega$ can be adjusted through the chirp and the delay between the two pulses. Most variants of SPIDER devices [16,19] rely on a sum frequency generation process in a nonlinear crystal, which constitutes a limiting factor for applications in the ultraviolet spectral range (UV). Indeed, it is challenging to find transparent crystals with the suitable phase matching conditions at wavelengths shorter than $400 \mathrm{~nm}$ in a second-order nonlinear optical process. Furthermore, if the wavelength of the pulse to be measured is below $380 \mathrm{~nm}$, the second-harmonic radiation would lie in the vacuum UV being absorbed by the oxygen of air. In spite of some d-scan [20] or FROG [21-23] variants for the characterization of UV pulses, the need of self-referenced and single-shot methods enabling measurement of weak pulses remains. To characterize UV pulses, some techniques adopt a frequency down-conversion process. The DC-SPIDER [24,25] relies, for instance, on the difference frequency generation (DFG) between the pulse to be characterized and a chirped pulse of smaller frequency. Nevertheless, this approach, as with other methods based on DFG such as DFG XFROG [22], requires two input beams at different frequencies, limiting its use to custom developments. Another solution is to exploit alternative nonlinear processes with higher orders of nonlinearity, as, for instance, degenerate four-wave mixing processes. Polarization gating (PG), self-diffraction (SD), or transient grating (TG) have thus been 
exploited in FROG variants [15]. Nevertheless, single-shot arrangements based on these processes suffer from geometrical smearing [26] because the signal does not propagate along the bisector of the beams. Furthermore, single-shot measurements of UV pulses by PG $[23,27]$ or TG [21] FROG need an input energy of a few tens or hundreds of $\mu \mathrm{J}$. A variant of SPIDER, the SD-SPIDER [28], operating at the fundamental frequency has also been reported, but the SD process is not phasematched and the device measures the phase of the second harmonic (and not the one of the pulse), implying an elaborate retrieval procedure. The use of a third-order nonlinear effect through spectral interferometry is nevertheless very attractive for a self-referenced and single-shot measurement of weak UV pulses. We have shown in this issue [14] the relevance of the rotational Doppler frequency shift $[7,10,11]$ enabling the production of two sheared replica of an unknown pulse at/near the original wavelength. The rotational Doppler shift (RDS) has been reported with waveplates in mechanical rotation [29] or with rotating molecules [10,11], but our approach relies on a third-order instantaneous electronic Kerr effect produced by pulses of twisted linear polarization (TLP). As shown in our previous work [14], the overall mechanism is perfectly phase-matched and provides a direct phase retrieval. It lifts, therefore, the inherent flaws of the SD phenomenon. The central purpose of the present paper is twofold. First, we provide a comprehensive analysis of the underlying effect. The origin of the Doppler shift and of the energy conservation is clarified from the third-order nonlinear polarization at play in our process. Second, the DEER-SPIDER method is evaluated under two different and rather challenging conditions: the characterization of short UV pulses ( 40 fs duration, $\lambda=350 \mathrm{~nm})$ and the characterization of longer IR pulses $(\lambda=800 \mathrm{~nm})$ tailored by a pulse shaper device. The production and characterization of the TLP pulse are described in detail.

\section{PRINCIPLE}

\section{A. Rotational Doppler effect}

The rotational Doppler effect can be seen as a variant of the well-known translational Doppler effect, which appears, for instance, when a wave is reflected on a moving object. The reflected (or counterpropagating) wave is frequency-shifted by $\Delta \omega=-2 \vec{k} \cdot \vec{v}$, with $\vec{k}$ the wave vector of the incident field and $\vec{v}$ the velocity of the object. The rotational Doppler effect for its part takes place, for instance, when a circularly polarized (CP) light interacts with an anisotropic object in rotation. It gives rise to a counter-rotating wave shifted by $\Delta \omega= \pm 2 \Omega_{\mathrm{rot}}, \Omega_{\mathrm{rot}}$ being the angular frequency of the object. The sign of the shift depends on the helicity of the CP wave with respect to the sense of rotation of the object. This effect can be readily understood by considering the example of the half-wave plate [29]. A half-wave plate rotates the polarization direction of a linearly polarized light by twice the angle between the incident polarization direction and the fast axis of the half-wave plate, namely from $\theta_{\text {in }}$ to $\theta_{\text {out }}=2 \theta_{\mathrm{pl}}-\theta_{\text {in }}$, with $\theta_{\mathrm{pl}}$ the angle of the fast axis. The half-wave plate flips, therefore, the helicity of a $\mathrm{CP}$ wave since at any time it produces the symmetrical of the electric field with respect to its fast axis $\left(\theta_{\text {in }}=-\omega t\right.$ becomes $\left.\theta_{\text {out }}=2 \theta_{\mathrm{pl}}+\omega t\right)$. A rotation of the fast axis of the wave plate by $\theta_{\mathrm{pl}}=\Omega_{\mathrm{rot}} t$ induces, therefore, a variation of the resulting field vector angular speed, namely of the angular frequency by $2 \Omega_{\text {rot }}$. It can be shown that a wave plate, other than a half-wave plate, produces a spectrally shifted counter-rotating wave together with an unshifted corotating wave. In a similar way, the translational Doppler effect with semitransparent objects gives rise to an unshifted transmitted wave in addition to the spectrally shifted reflected wave. The rotational Doppler shift (RDS) has been observed with anisotropic optical elements in mechanical rotation [29] or with rotating molecules $[10,11]$.

\section{B. Application to spectral shearing interferometry}

In SPIDER, two pulses delayed by $\tau$ and spectrally shifted by $\Omega$ interfere in a spectrometer. Letting the complex field of the pulse be $|E(\omega)| e^{i \varphi(\omega)}$ and the frequency-shifted pulse be $|E(\omega+\Omega)| e^{i \varphi(\omega+\Omega)}$, the measured interferogram will be written as [30]

$$
\begin{aligned}
S(\omega)= & |E(\omega)|^{2}+|E(\omega+\Omega)|^{2} \\
& +2|E(\omega)||E(\omega+\Omega)| \cos (\omega \tau+\Gamma(\omega)),
\end{aligned}
$$

where the spectral fringe pattern depends on $\Gamma(\omega)=\varphi(\omega+$ $\Omega)-\varphi(\omega)$, the spectral phase difference between the two pulses. The cosine argument can be extracted through Fourier filtering, and after subtracting the term $\omega \tau, \Gamma(\omega)$, which contains the phase $\varphi(\omega)$ to be recovered, can be isolated. The integration of $\Gamma(\omega)$ [16] provides (up to a constant) the spectral phase $\varphi(\omega)$ of the pulse

$$
\varphi(\omega) \approx \frac{1}{\Omega} \int_{0}^{\omega} \Gamma\left(\omega^{\prime}\right) d \omega^{\prime} .
$$

In standard SPIDER, the spectral shear is produced by nonlinear mixing of two time-delayed replicas of the pulse with a chirped pulse in a nonlinear crystal. The sheared pulses are centered near twice the carrier frequency of the pulse to be characterized, which limits the functionality for the characterization of UV laser pulses. To prevent this flaw, we exploit the rotational Doppler effect so as to produce two sheared replica at/near the original wavelength. For a suitable characterization by spectral-shearing interferometry, the frequency shear should be about a tenth of the spectral bandwidth, which corresponds typically to an angular speed of the anisotropy $\Omega_{\text {rot }}$ in the range of a few $\mathrm{rad} / \mathrm{ps}$. Such a rotating anisotropy being unreachable by a mechanical rotation, a different strategy must be adopted. Our approach takes advantage of a nonlinear interaction and more precisely on the third-order instantaneous electronic Kerr effect. This last manifests itself by the occurrence of a birefringence when a linearly polarized field interacts with a medium exhibiting a significant third-order susceptibility. When produced by a TLP pulse, the optical Kerr effect gives rise to a rotating anisotropy enabling the production of rotational Doppler shift. TLP pulses have already been exploited to induce unidirectional rotational motion of linear molecules [11] measured by the rotational Doppler effect. In the present case, the frequency shear $\Omega$ produced by the optical Kerr effect with TLP pulses can be retrieved by calculating the nonlinear polarization (see Appendix A) 


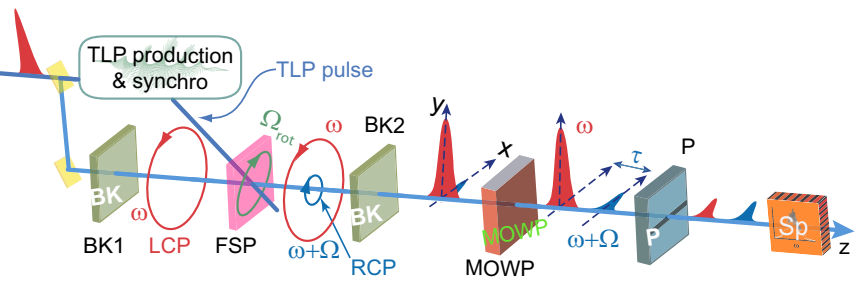

FIG. 1. Schematic representation of the DEER-SPIDER setup. BK, Berek compensator; FSP, fused silica plate; MOWP, multiple order wave plate; LCP (RCP), left (right) circular polarization; P, polarizer; $\mathrm{Sp}$, spectrometer.

revealing a counter-rotating $\mathrm{CP}$ field that reads

$$
E(t) \propto \chi^{(3)}\left|\varepsilon_{\mathrm{TLP}}(t)\right|^{2} \varepsilon(t) e^{i\left(\omega_{0} \pm 2 \Omega_{\mathrm{rot}}\right) t},
$$

with $\chi^{(3)}$ the third-order susceptibility, $\omega_{0}$ the central angular frequency, $\Omega_{\text {rot }}$ the angular frequency of the twisted polarization of the TLP pulse, and $\varepsilon_{\mathrm{TLP}}(t)$ and $\varepsilon(t)$ the complex field envelope of the TLP and the input pulse, respectively. As discussed in Appendix A 4, the frequency shift is concomitant to energy transfer from the characterized pulse to the TLP (or the reverse), ensuring the energy conservation.

\section{EXPERIMENTAL SETUP}

\section{A. DEER-SPIDER design}

The experimental setup for spectral shearing interferometry by the Doppler effect is depicted in Fig. 1.

The input beam is split into two arms using a reflection on a fused silica (FS) plate. The transmitted pulse is shaped in polarization in order to produce the TLP pulse as described in the next part. The other arm that contains the "reference" pulse (namely the field to be characterized) is circularly polarized using a $\mathrm{MgF} 2$ Berek compensator $\mathrm{BK} 1$ used as a quarter wave plate. Compared to zero-order quartz wave plates, the Berek compensators induces less dispersion (about $120 \mathrm{fs}^{2}$ at $350 \mathrm{~nm}$ ) and offers more flexibility since the dephasing can be adjusted as a function of the wavelength. Reference and TLP pulses, temporally synchronized, are focused by the same lens (focal length $f=15 \mathrm{~cm}$ ) into a $1 \mathrm{~mm}$ FS plate with a small angle. The TLP pulse induces a rotating birefringence in the plate so that after it, a spectrally shifted CP field of opposite handedness $E(\omega+\Omega)$ [or $E(\omega-\Omega)$ ] is produced together with a copropagating and stronger unshifted $\mathrm{CP}$ field of same helicity $E(\omega)$. The TLP pulse is blocked while the reference beam passes through a Berek compensator (BK 2) also used as a quarter wave plate, which transforms the two opposite $\mathrm{CP}$ fields into two crossed linear polarizations. The two sheared pulses are further time-delayed by a delay $\tau$ via a multiple order wave plate (MOWP), and their amplitudes are balanced by tuning the orientation of the polarizer $(\mathrm{P})$. Finally, the beam is sent into a spectrometer to measure the interferogram. The delay $\tau$ is produced by a $1.65-\mathrm{mm}$-thick Calcite plate for the characterization of short UV pulses $(\tau=1.45 \mathrm{ps})$ and by a 5-mm-thick Calcite plate for the characterization of long and shaped IR pulses ( $\tau=3 \mathrm{ps})$.

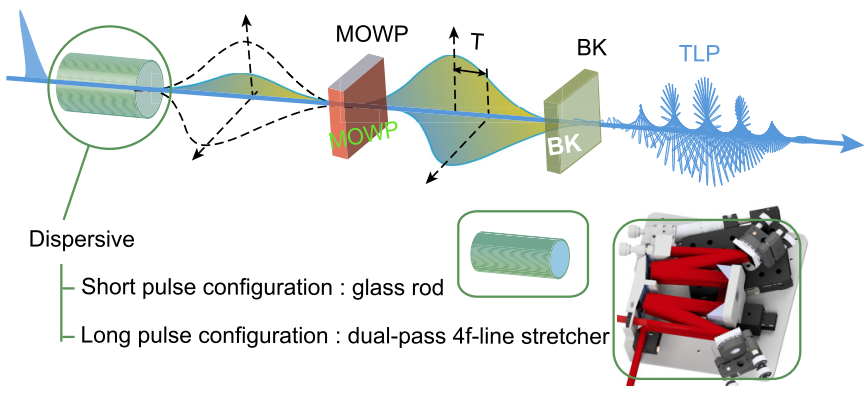

FIG. 2. TLP pulse generation: MOWP, multiple order wave plate; BK, Berek compensator; TLP, pulse of twisted linear polarization.

\section{B. Pulse of twisted linear polarization: Production and characterization}

Pulses of twisted linear polarization can be produced in different ways [31]. The method selected here relies on the production of two counter-rotating $\mathrm{CP}$ and time-delayed chirped pulses. A TLP pulse can be seen as the superposition of two counter-rotating $\mathrm{CP}$ fields with slightly different frequencies. This point is readily understood by writing the TLP pulse with two in-phase components along $x$ and $y$ exhibiting a continuous variation of their relative amplitudes through a sinusoidal temporal dependence:

$$
\vec{E}=\varepsilon(t) e^{i \omega_{0} t}\left(\begin{array}{c}
\sin \left(\Omega_{\mathrm{rot}} t\right) \\
\cos \left(\Omega_{\mathrm{rot}} t\right)
\end{array}\right) .
$$

The field vector of such a TLP pulse rotates with a period $T_{\text {rot }}=2 \pi / \Omega_{\text {rot }}$, and the previous equation can then be modified as follows:

$$
\begin{aligned}
\vec{E} & =\frac{\varepsilon(t) e^{i \omega_{0} t}}{2}\left(\begin{array}{c}
-i e^{i \Omega_{\mathrm{rot}} t}+i e^{-i \Omega_{\mathrm{rot}} t} \\
e^{i \Omega_{\mathrm{rot}} t}+e^{-i \Omega_{\mathrm{rot}} t}
\end{array}\right) \\
& =\frac{\varepsilon(t) e^{i\left(\omega_{0} t+\Omega_{\mathrm{rot}} t\right)}}{2}\left(\begin{array}{c}
-i \\
1
\end{array}\right)+\frac{\varepsilon(t) e^{i\left(\omega_{0} t-\Omega_{\mathrm{roo}} t\right)}}{2}\left(\begin{array}{l}
i \\
1
\end{array}\right)
\end{aligned}
$$

corresponding to two $\mathrm{CP}$ fields of opposite handedness and frequency-shifted by $\delta \omega=2 \Omega_{\mathrm{rot}}=4 \pi / T_{\mathrm{rot}}$. Our polarization shaping, depicted in Fig. 2, is based on this principle. It uses a sequence of two chirped pulses temporally delayed by $T$, $T$ being smaller than the pulse duration so as to ensure an overlap between the two pulses. In this purpose, the pulse is first chirped using a dispersive medium for the UV configuration or a dual-pass $4 f$-line stretcher for the long pulse IR configuration. The chirped pulse is then transformed in two time-delayed perpendicularly polarized components using a MOWP, and the two components are circularly polarized with opposite handedness by means of a Berek compensator acting as a quarter wave plate. Both pulses present an instantaneous frequency that varies linearly with the time: $\omega(t)=\omega_{0}+2 a t$, where the expression of $a$ for a Gaussian pulse is

$$
a=\frac{\Phi^{\prime \prime}}{\left(\frac{\Delta t_{\mathrm{FTL}}^{2}}{2 \sqrt{2} \ln 2}\right)^{2}+2 \Phi^{\prime \prime 2}},
$$

with $\Delta t_{\mathrm{FTL}}$ the full width at half-maximum duration of the Fourier transform limited (FTL) pulse, and $\Phi^{\prime \prime}$ is the quadratic spectral phase introduced with the chirp. The two pulses, 


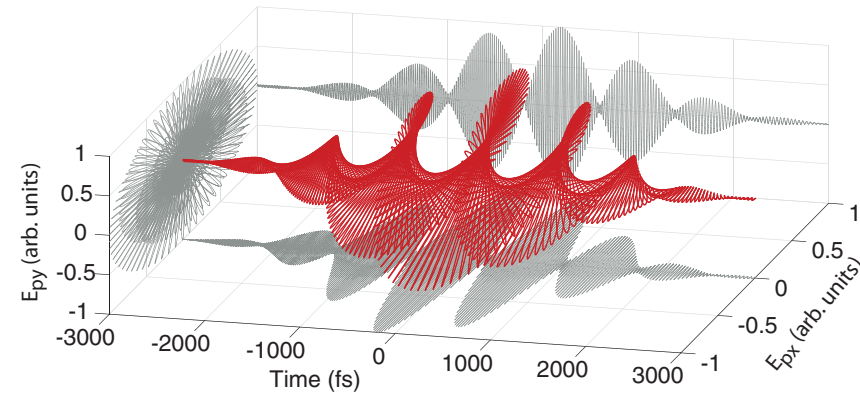

FIG. 3. Representation of the TLP electric field profile for $\lambda=$ $350 \mathrm{~nm}$. The parameters used for the calculation are the experimental parameters for the case 1 (see text), except for the central frequency that is fixed to $\omega_{0} / 20$ for a better visualization.

delayed by $T$, presents therefore a frequency shift versus time $\delta \omega(t)=\omega(t+T)-\omega(t)=2 a T$, which is constant. Since the two pulses are circularly polarized with opposite handedness, the resulting field is linearly polarized with a rotating polarization of period $T_{\mathrm{rot}}=4 \pi / \delta \omega=2 \pi / a T$. The expected Doppler shift with such a TLP pulse is $\Omega=2 \Omega_{\text {rot }}=4 \pi / T_{\text {rot }}$. Compared to other methods [11], the TLP pulse produced in this way can be longer with possibly many turns of the electric field (see, for instance, Fig. 3). Long TLP pulses feature the advantage of being less sensitive to the input chirp enabling the characterization of larger spectral phases. The temporal synchronization between the TLP pulse and the reference pulse is also less critical.

The first configuration (called hereafter case 1) is the one implemented for characterizing short UV pulses $(\lambda=$ $350 \mathrm{~nm}$ ). The main parameters of the TLP pulse production are $\Delta t_{\mathrm{FTL}}=40 \mathrm{fs}, \Phi^{\prime \prime} \approx 24000 \mathrm{fs}^{2}$, and $T=190 \mathrm{fs}$. From the above discussion, the rotational period of spinning polarization is expected to be equal to $T_{\text {rot }}=1.6 \mathrm{ps}$ and the shear to $\Omega=7.9 \mathrm{rad} / \mathrm{ps}$. A representation of the TLP electric field profile is depicted in Fig. 3.

To characterize the TLP pulse, one can exploit the electronic Kerr effect (or polarization gating) with the same experimental scheme as in Fig. 1 but using a linearly polarized reference beam and a linear analyzer (by tuning BK1 and BK2). By varying the delay between the reference and TLP pulses, the measured Kerr signal, corresponding to the depolarization experienced by the reference pulse, is maximum when the polarization of the pump and the reference form an angle of $45^{\circ}$. A modulation with a periodicity of $T_{\text {rot }} / 4$ is therefore expected. A typical measurement of the Kerr signal is depicted in Fig. 4. A modulation of period $\Delta t_{\text {mod }} \approx 0.4 \mathrm{ps}$ is observed indicating a TLP rotational period $T_{\mathrm{rot}} \approx 1.6 \mathrm{ps}$ in good agreement with the parameters used for the polarization shaping. The shear has also been evaluated by measuring the spectrum of the field when produced with TLP pulses of opposite rotating polarization so as to shift the observed signal from $\Omega$ to $-\Omega$ (which is a bit more accurate than the difference between shifted and unshifted pulse). The polarizer in Fig. 1 is set horizontally to measure only the component shifted by the Doppler effect. Two spectra have then been measured by rotating by $90^{\circ}$ the BK in Fig. 2 in order to reverse the twisting polarization of the TLP pulse.

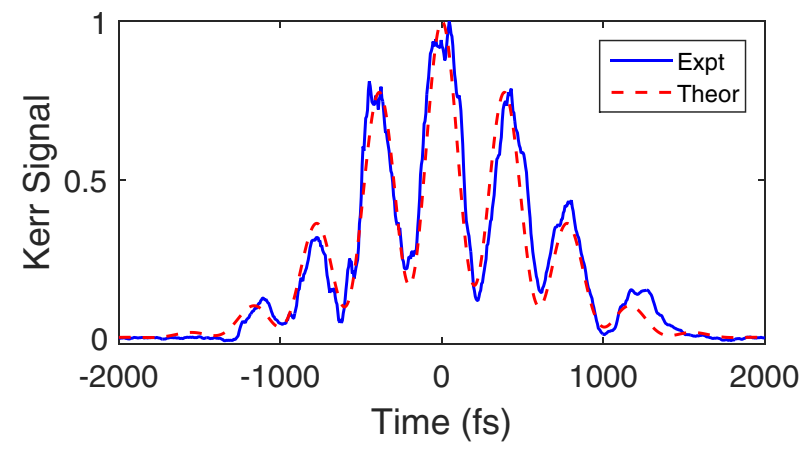

FIG. 4. Characterization of the TLP pulse by Kerr gating for the UV configuration (case 1; see the text). The calculation is performed with a TLP pulse of rotational period $T_{\text {rot }}=1.6 \mathrm{ps}$.

The two spectra shown in Fig. 5 are found to be shifted by $2 \Omega=16 \mathrm{rad} / \mathrm{ps}$ close to the value expected from the angular speed of the TLP pulse. We point out that the spectra perfectly overlap with each other when shifted by $2 \Omega$, meaning that the shear is obtained without any noticeable distortions of the spectrum.

The second configuration investigated in the present work is dedicated to the characterization of long IR and shaped pulses $\left(\Delta t_{\mathrm{FTL}}=100 \mathrm{fs}\right.$ and $\left.\lambda=800 \mathrm{~nm}\right)$. In this case (called case 2), the small spectral bandwidth and the wavelength of the pulse prevent the use of a dispersive medium to induce a sufficient chirp of the TLP pulse. Instead, the pulse is stretched using a double-pass $4 f$-line reflected pulse stretcher inducing a quadratic spectral phase $\Phi^{\prime \prime}=160000 \mathrm{fs}^{2}$. The duration of each chirped pulse is $\Delta t_{\mathrm{ch}}=4.4 \mathrm{ps}$ and the delay is $T=$ $500 \mathrm{fs}$ so that the rotational period of the spinning polarization is $T_{\text {rot }}=4$ ps providing a shear $\Omega=3.14 \mathrm{rad} / \mathrm{ps}$.

\section{EXPERIMENTAL RESULT}

\section{A. Characterization of UV pulses}

The UV pulse $\left(\Delta t_{\mathrm{FTL}}=40 \mathrm{fs}, \lambda=350 \mathrm{~nm}\right)$ is delivered by a Ti:sapphire laser equipped with a NOPA (noncollinear optical parametric amplifier). The input pulse of typical energy $2 \mu \mathrm{J}$ is characterized by our DEER-SPIDER setup. The interferogram, obtained by tuning the orientation of the

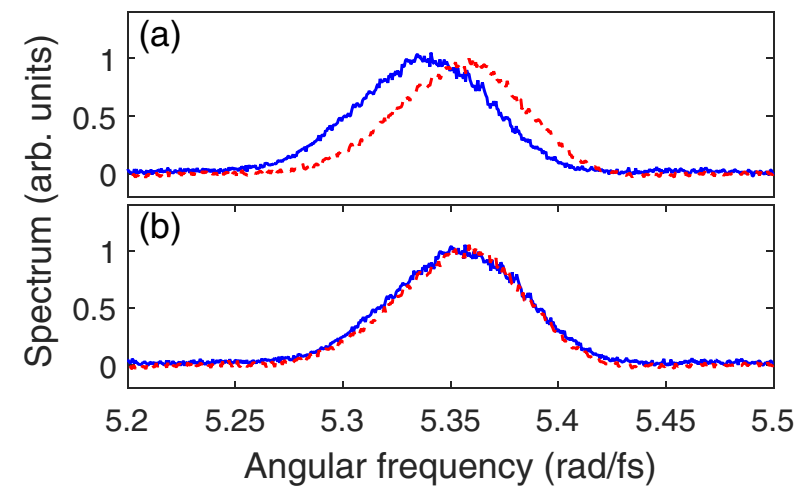

FIG. 5. (a) Spectrum of the UV pulse for opposite twisting polarization of the TLP pulse; (b) same as (a) by shifting the blue curve by $2 \Omega$. 

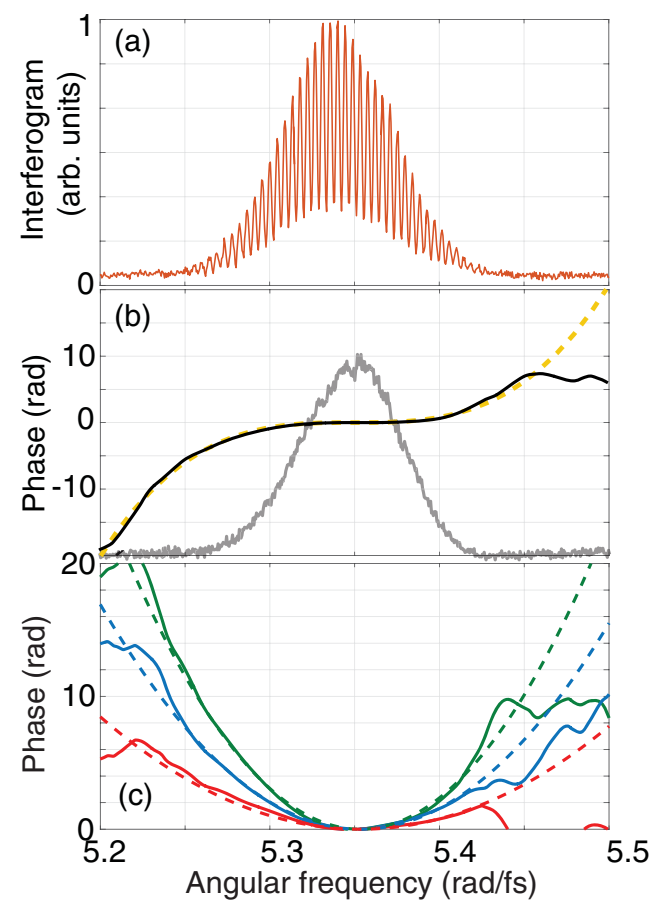

FIG. 6. (a) Interferogram. (b) Spectral phases of a UV pulse retrieved from the DEER-SPIDER (black line) with the laser spectrum (gray line). (c) Spectral phase induced by different fused silica (FS) plates: the red (lower), blue (middle), and green (upper) line denote 6,12 , and $18 \mathrm{~mm}$ thicknesses. Measurements in solid lines are compared to the FS second-order dispersion in dashed lines.

polarizer (P) in Fig. 1 so as to maximize the contrast of the spectral fringes, is depicted in Fig. 6(a). The spectral phase is encoded into the fringes pattern and can be retrieved using the inversion procedure given in Eq. (2) but with a minor modification for a better accuracy (see Appendix B 1). Figure 6(b) depicts the DEER-SPIDER characterization of the pulse delivered by the laser system. A small resulting cubic phase can be clearly distinguished. The latter can be imputed to an effect of dispersion mismatch undergone by the two sheared pulses. Standard SPIDER designs are known to suffer from a dispersion mismatch introduced by the beamsplitter used to produce the pair of pulses. In the present DEER-SPIDER, the phase mismatch originates from the differential dispersion induced by the MOWP in Fig. 1 since the pulse separation is performed after the interaction. The differential quadratic phase induced in this way (about $280 \mathrm{fs}^{2}$ ) manifests as an additional cubic contribution in the retrieved phase after the inversion procedure. This contribution, depicted in Fig. 6(b) as a dashed line, reveals that the initial phase can be entirely attributed to this artifact. While it can be accurately assessed and subtracted, we point out that this effect could also be significantly reduced by the use of MgF2 MOWP, which induces a differential dispersion 3.6 times weaker than calcite for a given delay $\tau$. Furthermore, as discussed in the Conclusion, the implementation of a homodyne detection would overcome this problem. The reliability of our method has been further checked by inducing known chirps by means of fused silica (FS) plates of different thicknesses. After subtracting the initial phase, the phases attributed to the FS plates can be
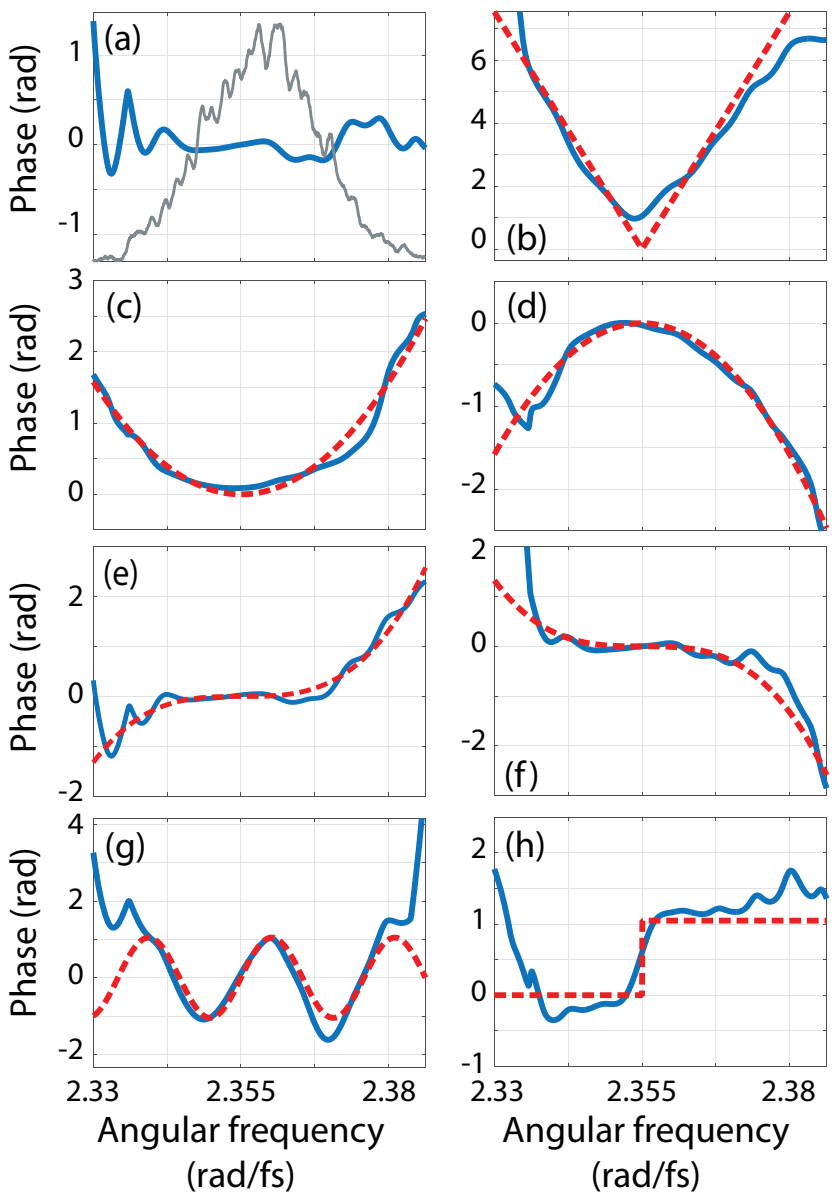

FIG. 7. Characterization of various spectral phases $\varphi(\omega)$ applied with the pulse shaper: (a) unshaped (with the laser spectrum shown by a gray line), (b) triangular phase function of \pm 300 fs slope, (cd) $\varphi(\omega)= \pm \phi^{(2)} / 2\left(\omega-\omega_{0}\right)^{2}$ with $\phi^{(2)}=5 \times 10^{3} \mathrm{fs}^{2}$, (e-f) $\varphi(\omega)=$ $\pm \phi^{(3)} / 6\left(\omega-\omega_{0}\right)^{3}$ with $\phi^{(3)}=5 \times 10^{5} \mathrm{fs}^{3},(\mathrm{~g}) \varphi(\omega)=\pi / 3 \sin (\omega \tau)$ with $\tau=300 \mathrm{fs}$, (h) $\pi / 3$ phase jump. The red dashed lines depict the phase encoded with the pulse shaper, and the blue lines depict the phase retrieved by our device.

measured, and the result shown in Fig. 6(c) reveals a quadratic spectral phase of increasing amplitude. Those phases have then been compared with the prediction from second-order dispersion and were found in very good agreement within an RMS error field $\epsilon \leqslant 0.1$. These observations highlight the ability of our method to provide a reliable characterization of ultrafast UV laser sources on a single-shot basis.

\section{B. Characterization of IR pulses}

As an additional test of our method, the ability to retrieve different types of spectral phases is investigated. A laser pulse delivered by a Ti:sapphire laser $\left(\Delta t_{\mathrm{FTL}}=100 \mathrm{fs}\right.$ and $\lambda=$ $800 \mathrm{~nm}$ ) is spectrally phase-shaped via a standard pulse shaper device using a programmable 1D dual mask LC-SLM array (SLM-320d from Jenoptik), and the applied spectral phases are compared to the measured ones. A description of the pulse shaper arrangement can be found in Ref. [32]. Various phase modulations have been applied with the pulse shaper. The main results, summarized in Fig. 7, demonstrate the ability 
of our device to retrieve different spectral phases with a great fidelity. As shown, standard cubic or quadratic spectral phases have been characterized (with their signs) together with more exotic modulations such as triangular or sinusoidal phase functions. The result depicted in Fig. 7 corresponds to the direct retrieved phases without any correction from the phase mismatch that is negligible in the present case. We emphasize that the chirp to be characterized should remain small enough to keep the main features of the TLP pulse unchanged in order to preserve the Doppler shift. This condition is fulfilled for all phases except maybe for the phase step [Fig. 7(h)]. As discussed in Appendix B 2, both the spectral phase and the amplitude of the field produced by the Doppler effect are altered with this shaping. Nevertheless, the deterioration of the transcribed phase remains moderate so that the phase retrieval remains satisfactory considering that the phase step is notoriously difficult to characterize. While this limitation is inherent to any SPIDER design and to most self-referenced characterization methods, the ability of the present device to characterize such a set of significantly different phases confirms the relevance of our DEER method. Furthermore, the use of alternative strategies for producing the TLP pulse could provide a Doppler effect even more immune with respect to the input phase. The TLP pulse could, for instance, be produced by adding two counter-rotating $\mathrm{CP}$ fields with slightly different frequencies through the selection of two narrow sidebands within the spectrum. This could enable a better characterization of phases with abrupt variations or jumps but at the expense of the energy consumed in the shaping process.

\section{CONCLUSION AND DISCUSSION}

This paper provides an extended discussion and substantiation of the recently reported DEER-SPIDER method [14]. The major innovation relies on the use of the rotational Doppler effect enabling the production of a frequency shear in the absence of frequency up-conversion. This nonconventional approach provides an extended functionality toward the UV spectral domain. The method has been evaluated with two different and rather challenging conditions: characterization of IR shaped pulses and of UV pulses. In both cases, the performance of the setup, in terms of phase reconstruction, turns out to be of high quality and confirms the relevance of the method. In particular, the DEER-SPIDER method proves to be well suited for the single-shot characterization of UV ultrafast laser sources, which is of great interest for several applications, as, for instance, in the context of XFEL seeding. The rotational Doppler effect as implemented here could also be exploited for other applications, as, for instance, to control the wavelengths of $\mathrm{THz}$ fields not otherwise easily accessible. Several improvements could be made to the device. For instance, instead of using a BK compensator to circularly polarize the reference pulse, one could exploit the depolarization induced by metallic reflection so as to limit the dispersion or enable operation in the deep UV. Standard SPIDER designs are also affected by the dispersion mismatch introduced by the beamsplitter used to produce the pair of pulses. This effect, limited in the DEER-SPIDER since the pulse separation is performed after the interaction, could be significantly reduced by using a MgF2 MOWP for which the dispersion mismatch would be of $50 \mathrm{fs}^{2}$ and $5 \mathrm{fs}^{2}$ at $350 \mathrm{~nm}$ for a delay $\tau=1 \mathrm{ps}$ and $100 \mathrm{fs}$, respectively. Finally, the present device can readily give access to a homodyne detection [33] so as to overcome the calibration issue of the delay $\tau$ in Eq. (1). Any miscalibration of this delay will indeed result in a systematic quadratic error in the retrieved spectral phase. To get rid of it, two measurements with opposite rotating polarizations of the TLP pulse could be conducted, providing a spectral phase difference equal to $\omega \tau+\varphi(\omega+\Omega)-\varphi(\omega)$ or $\omega \tau+\varphi(\omega-\Omega)-\varphi(\omega)$. By subtracting the two quantities, one would enable a phase retrieval without any precalibration of the delay $\tau$ and insensitive to the dispersion mismatch introduced by the MOWP.

\section{ACKNOWLEDGMENTS}

This work was supported by the CNRS, the FEMTO network, the ERDF Operational Programme-Burgundy, and the EIPHI Graduate School (Contract No. ANR-17-EURE-0002). The Ph.D. of E.S. is funded by a CIFRE contract (2018/0253) granted by ANRT. Calculations were performed using HPC resources from DNUM-CCUB (Université de Bourgogne).

\section{APPENDIX A: THEORETICAL ANALYSIS OF THE PRODUCED SHEAR}

The objective of this Appendix is to clarify the origin of the sheared pulse from the third-order nonlinear polarization $\vec{P}(t)$ at play in the DEER process.

\section{Nonlinear polarization}

Let us first consider the very general case of two beams crossing at a small angle in an isotropic medium consisting of a thin piece of glass in the $(x, y)$ plane (see Fig. 1 of the main text). The first pulse, called hereafter the "pump pulse," has a complex amplitude written as

$$
\vec{E}_{\mathrm{p}}=e^{i\left(\omega_{0} t-\vec{k}_{\mathrm{p}} \cdot \vec{r}\right)}\left(\begin{array}{l}
\varepsilon_{\mathrm{p}_{x}}(t) \\
\varepsilon_{\mathrm{p}_{\mathrm{y}}}(t)
\end{array}\right)
$$

while the second one called "probe pulse" is of the form

$$
\vec{E}=e^{i\left(\omega_{0} t-\vec{k} \cdot \vec{r}\right)}\left(\begin{array}{l}
\varepsilon_{x}(t) \\
\varepsilon_{y}(t)
\end{array}\right),
$$

where the time-dependent amplitudes along $x$ and $y$ of both fields can be complex. The signal of interest is produced by a third-order nonlinear optical process at the fundamental angular frequency $\omega_{0}$ in the direction of the probe beam propagation so that the conservation of the wave vector imposes a field produced with the wave vector $\vec{k}=\vec{k}_{\mathrm{p}}-\vec{k}_{\mathrm{p}}+\vec{k}$ (implying a sequence of fields $E_{\mathrm{p}} E_{\mathrm{p}}^{*} E$ ). This effect corresponds to the cross-coupling electronic Kerr effect with the pump pulse inducing modifications of the optical properties of the medium experienced by the (weaker) probe pulse. For calculating the part of the nonlinear polarization that influences the probe pulse, we will assume that the system responds instantaneously to the applied field so that the nonlinear polarization can be described in the time domain (and that Kleinman's symmetry is valid). We also use the properties of symmetry of the third-order susceptibility tensor for isotropic materials 
$\left(\chi_{i j k l}^{(3)}\right.$ with $i, j, k, l=x, y$, or $z$ ) that comprises 21 nonzero elements of which only 3 are independent. Under these conditions, the nonlinear polarization envelope can be reduced to the following expression:

$$
\left(\begin{array}{l}
P_{x}(t) \\
P_{y}(t)
\end{array}\right)=\frac{\epsilon_{0} \chi^{(3)}}{3}\left(\begin{array}{ll}
P_{x x}(t) & P_{x y}(t) \\
P_{y x}(t) & P_{y y}(t)
\end{array}\right)\left(\begin{array}{l}
\varepsilon_{x}(t) \\
\varepsilon_{y}(t)
\end{array}\right),
$$

where $\chi^{(3)}=\chi_{x x x x}^{(3)}=\chi_{y y y y}^{(3)}$, and

$$
\begin{aligned}
& P_{x x}=6\left|\varepsilon_{\mathrm{p}_{x}}(t)\right|^{2}+2\left|\varepsilon_{\mathrm{p}_{y}}(t)\right|^{2}, \\
& P_{y y}=6\left|\varepsilon_{\mathrm{p}_{y}}(t)\right|^{2}+2\left|\varepsilon_{\mathrm{p}_{x}}(t)\right|^{2}, \\
& P_{x y}=P_{y x}=2\left(\varepsilon_{\mathrm{p}_{x}}(t) \varepsilon_{\mathrm{p}_{y}}^{*}(t)+\varepsilon_{\mathrm{p}_{x}}^{*}(t) \varepsilon_{\mathrm{p}_{y}}(t)\right) .
\end{aligned}
$$

\section{Circular polarizer and analyzer for the probe pulse}

We consider for the probe pulse (the pulse to be characterized in the DEER-SPIDER setup) a left-handed or counterclockwise circularly polarized (CP) field written as

$$
\left(\begin{array}{l}
\varepsilon_{x}(t) \\
\varepsilon_{y}(t)
\end{array}\right)=\varepsilon(t)\left(\begin{array}{l}
i \\
1
\end{array}\right)
$$

After the interaction, a circular analyzer is used to isolate the component of opposite handedness so that the signal field envelope will be given by

$$
\begin{aligned}
\varepsilon_{\text {sig }}(t) & \propto i P_{y}(t)-P_{x}(t) \\
& \propto \chi^{(3)} \varepsilon(t)\left[\left(\left|\varepsilon_{\mathrm{p}_{y}}(t)\right|^{2}-\left|\varepsilon_{\mathrm{p}_{x}}(t)\right|^{2}\right) i-\frac{P_{x y}}{2}\right] .
\end{aligned}
$$

\section{Results for an "ideal" pulse of twisted linear polarization}

To gain more insight into the produced shear, we consider for the pump field the case of an "ideal" TLP pulse defined in Eq. (4) of the main text. The amplitudes in Eq. (A1), therefore, give

$$
\left(\begin{array}{c}
\varepsilon_{\mathrm{p}_{x}}(t) \\
\varepsilon_{\mathrm{p}_{y}}(t)
\end{array}\right)=\varepsilon_{\mathrm{p}}(t)\left(\begin{array}{c}
\sin \left(\Omega_{\mathrm{rot}} t\right) \\
\cos \left(\Omega_{\mathrm{rot}} t\right)
\end{array}\right) .
$$

The two components along $x$ and $y$ are in phase at any time but with a continuous variation of their relative amplitudes giving rise to the desired field of spinning polarization (that rotates here in the clockwise direction). The real and imaginary parts of Eq. (A6) can be rewritten according to the expression of the TLP pulse, giving

$$
\begin{aligned}
\left|\varepsilon_{\mathrm{p}_{y}}(t)\right|^{2}-\left|\varepsilon_{\mathrm{p}_{x}}(t)\right|^{2} & =\left|\varepsilon_{\mathrm{p}}(t)\right|^{2}\left(\cos ^{2}\left(\Omega_{\mathrm{rot}} t\right)-\sin ^{2}\left(\Omega_{\mathrm{rot}} t\right)\right) \\
& =\left|\varepsilon_{\mathrm{p}}(t)\right|^{2} \cos \left(2 \Omega_{\mathrm{rot}} t\right)
\end{aligned}
$$

and

$$
\begin{aligned}
\frac{P_{x y}}{2} & =\varepsilon_{\mathrm{p}_{x}}(t) \varepsilon_{\mathrm{p}_{y}}^{*}(t)+\varepsilon_{\mathrm{p}_{x}}^{*}(t) \varepsilon_{\mathrm{p}_{y}}(t) \\
& =2\left|\varepsilon_{\mathrm{p}}(t)\right|^{2} \sin \left(\Omega_{\mathrm{rot}} t\right) \cos \left(\Omega_{\mathrm{rot}} t\right) \\
& =\left|\varepsilon_{\mathrm{p}}(t)\right|^{2} \sin \left(2 \Omega_{\mathrm{rot}} t\right) .
\end{aligned}
$$

Equation (A6) is then modified as follows:

$$
\begin{aligned}
\varepsilon_{\mathrm{sig}}(t) & \propto i \chi^{(3)}\left|\varepsilon_{\mathrm{p}}(t)\right|^{2} \varepsilon(t)\left[\cos \left(2 \Omega_{\mathrm{rot}} t\right)+i \sin \left(2 \Omega_{\mathrm{rot}} t\right)\right] \\
& \propto i \chi^{(3)}\left|\varepsilon_{\mathrm{p}}(t)\right|^{2} \varepsilon(t) e^{2 i \Omega_{\mathrm{rot}} t},
\end{aligned}
$$

providing a signal field equal to

$$
E_{\mathrm{sig}}(t) \propto i \chi^{(3)}\left|\varepsilon_{\mathrm{p}}(t)\right|^{2} \varepsilon(t) e^{i\left(\omega_{0}+2 \Omega_{\mathrm{rot}}\right) t} .
$$

The frequency shear appears therefore explicitly from the previous equation. As expected, the pulse is spectrally shifted by $\Delta \omega=2 \Omega_{\text {rot }}$, i.e., twice the angular frequency of the spinning polarization. Furthermore, the shift is toward higher frequencies since the probe $\mathrm{CP}$ field rotates in the counterclockwise direction, i.e., in the opposite direction as compared to the polarization of the TLP pulse (clockwise). If the direction of rotation of the TLP pulse is reversed, $\Omega_{\text {rot }}$ becomes $-\Omega_{\text {rot }}$ and the field then becomes redshifted. We emphasize that for a continuous TLP pulse, or for a TLP pulse long enough compared to $\varepsilon(t)$, the field produced in this way [Eq. (A11)] is the exact frequency-shifted replica of the input probe pulse. It can be recalled that in the experimental configurations of our work, the duration of the TLP pulse is much longer than the pulse to characterize.

While the previous streamlined approach features the advantage to provide a simple analytic expression, a thorough analysis reproducing more closely the experimental conditions is of interest in order to assess the reliability and limits of the method.

\section{Propagation equation}

Neglecting the linear dispersion terms and expressing the fields $\varepsilon_{x, y}$ so that $\left|\varepsilon_{x, y}\right|^{2}$ correspond to the intensity for the horizontal (vertical) polarization, one obtains the following propagation coupled equations for the field to characterize:

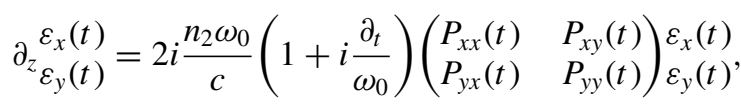

where $n_{2}=\frac{4}{3} \frac{\chi^{(3)}}{\epsilon_{0} c n_{0}^{2}}$ is the nonlinear refractive index of the material, and

$$
\begin{aligned}
& P_{x x}=\left|\varepsilon_{\mathrm{p}_{x}}(t)\right|^{2}+\frac{1}{3}\left|\varepsilon_{\mathrm{p}_{y}}(t)\right|^{2}, \\
& P_{y y}=\left|\varepsilon_{\mathrm{p}_{x}}(t)\right|^{2}+\frac{1}{3}\left|\varepsilon_{\mathrm{p}_{y}}(t)\right|^{2}, \\
& P_{x y}=P_{y x}=\frac{1}{3}\left(\varepsilon_{\mathrm{p}_{x}}(t) \varepsilon_{\mathrm{p}_{y}}^{*}(t)+\varepsilon_{\mathrm{p}_{x}}^{*}(t) \varepsilon_{\mathrm{p}_{y}}(t)\right) .
\end{aligned}
$$

The same kind of coupled propagation equations drive the propagation of the TLP pulse:

$$
\partial_{z}^{\varepsilon_{\mathrm{p}_{x}}(t)}=2 i \frac{n_{2} \omega_{0}}{c}\left(1+i \frac{\partial_{t}}{\omega_{0}}\right)\left(\begin{array}{ll}
P_{\mathrm{p}_{x}}(t) & P_{\mathrm{p}_{x y}}(t) \\
P_{\mathrm{p}_{y x}}(t) & P_{\mathrm{p}_{y y}}(t)
\end{array}\right){ }_{\varepsilon_{\mathrm{p}_{y}}(t)}^{\varepsilon_{\mathrm{p}_{x}}(t)},
$$

with

$$
\begin{aligned}
& P_{\mathrm{p}_{x x}}=\left|\varepsilon_{x}(t)\right|^{2}+\frac{1}{3}\left|\varepsilon_{y}(t)\right|^{2}, \\
& P_{\mathrm{p}_{y y}}=\left|\varepsilon_{x}(t)\right|^{2}+\frac{1}{3}\left|\varepsilon_{y}(t)\right|^{2}, \\
& P_{\mathrm{p}_{x y}}=P_{\mathrm{p}_{y x}}=\frac{1}{3}\left(\varepsilon_{x}(t) \varepsilon_{y}^{*}(t)+\varepsilon_{x}^{*}(t) \varepsilon_{y}(t)\right) .
\end{aligned}
$$

It is interesting to compare the present physical situation to the one taking place with the rotational Doppler effect induced by a wave plate or a molecular wave packet in rotation. During the latter processes, the circularly polarized electromagnetic field applies a torque to the object. This torque then induces a change of the energy of the rotating body and leads, by the conservation of the angular momenta, to the frequency shift 
of the electromagnetic field. In other words, in the latter situation, the frequency shift is concomitant to an energy transfer from the electromagnetic field to the rotating body (i.e., the plate or the molecules). In the present case, such an argument cannot be invoked, since the anisotropy is not produced by a physical rotating object but through a nonlinear interaction in which the matter acts only as an intermediary between the TLP and the pulse to characterize. From the above propagation equations, one can calculate the energy variation of the pulse to be characterized all along the propagation. After some cumbersome but straightforward calculations, one obtains the evolution of the energy $\mathcal{E}(z)=\int\left|\varepsilon_{x}\right|^{2}+\left|\varepsilon_{y}\right|^{2} d t$ :

$$
\partial_{z} \mathcal{E} \propto-\frac{n_{2}}{c} \int\left(\varepsilon_{x} \varepsilon_{y}^{*}+\varepsilon_{x}^{*} \varepsilon_{y}\right) \partial_{t}\left(\varepsilon_{\mathrm{p}_{x}} \varepsilon_{\mathrm{p}_{y}}^{*}+\varepsilon_{\mathrm{p}_{x}}^{*} \varepsilon_{\mathrm{p}_{y}}\right) d t,
$$

while the evolution of the TLP energy $\mathcal{E}_{\mathrm{p}}(z)=\int\left|\varepsilon_{\mathrm{p}_{x}}\right|^{2}+$ $\left|\varepsilon_{\mathrm{p}_{y}}\right|^{2} d t$ is given by

$$
\partial_{z} \mathcal{E}_{\mathrm{p}} \propto-\frac{n_{2}}{c} \int\left(\varepsilon_{\mathrm{p}_{x}} \varepsilon_{\mathrm{p}_{y}}^{*}+\varepsilon_{\mathrm{p}_{x}}^{*} \varepsilon_{\mathrm{p}_{y}}\right) \partial_{t}\left(\varepsilon_{x} \varepsilon_{y}^{*}+\varepsilon_{x}^{*} \varepsilon_{y}\right) d t .
$$

Integrating by parts the latter equation, one then obtains that there is an energy transfer from the pulse to characterize to the pump (or the reverse depending on the relative direction of rotation of the TLP with respect to the circularity of the pulse) without any energy transfer to the nonlinear medium:

$$
\partial_{z} \mathcal{E}_{\mathrm{p}}=-\partial_{z} \mathcal{E}
$$

It is also interesting to examine the photon number conservation of the characterized pulse during the nonlinear process. For this, it is easier to express the coupled propagation equations in the circularly polarization basis. This is done by defining a new set of electric fields:

$$
\begin{aligned}
& \varepsilon_{ \pm}=\frac{\varepsilon_{x} \pm i \varepsilon_{y}}{\sqrt{2}}, \\
& \varepsilon_{\mathrm{p}_{ \pm}}=\frac{\varepsilon_{\mathrm{p}_{x}} \pm i \varepsilon_{\mathrm{p}_{y}}}{\sqrt{2}} .
\end{aligned}
$$

In this basis, the propagation of the pulse to characterize is

$$
\partial_{z_{-}(t)}^{\varepsilon_{+}(t)}=4 i \frac{n_{2} \omega_{0}}{c}\left(1+i \frac{\partial_{t}}{\omega_{0}}\right)\left(\begin{array}{ll}
P_{++}(t) & P_{+-}(t) \\
P_{-+}(t) & P_{--}(t)
\end{array}\right) \varepsilon_{\varepsilon_{-}}(t),
$$

where

$$
\begin{aligned}
& P_{++}(t)=P_{--}(t)=\left|\varepsilon_{\mathrm{p}_{+}}\right|^{2}+\left|\varepsilon_{\mathrm{p}_{-}}\right|^{2}, \\
& P_{+-}(t)=P_{-+}^{*}(t)=\varepsilon_{\mathrm{p}_{+}} \varepsilon_{\mathrm{p}_{-}}^{*} .
\end{aligned}
$$

For a perfect TLP pulse (i.e., far longer than the pulse to characterize), one has

$$
P_{++}(t)=P_{--}(t)=\left|\varepsilon_{\mathrm{p}_{+}}\right|^{2}+\left|\varepsilon_{\mathrm{p}_{-}}\right|^{2} \simeq I_{\mathrm{p}},
$$

where $I_{\mathrm{p}}$ is the total intensity of the TLP that can be considered as time-independent (at least, over the characterized pulse duration). Moreover, one has

$$
\begin{aligned}
& P_{+-}(t)=I_{\mathrm{p}} / 2 e^{2 i \Omega_{\mathrm{rot}} t}, \\
& P_{-+}(t)=I_{\mathrm{p}} / 2 e^{-2 i \Omega_{\mathrm{rot}} t} .
\end{aligned}
$$

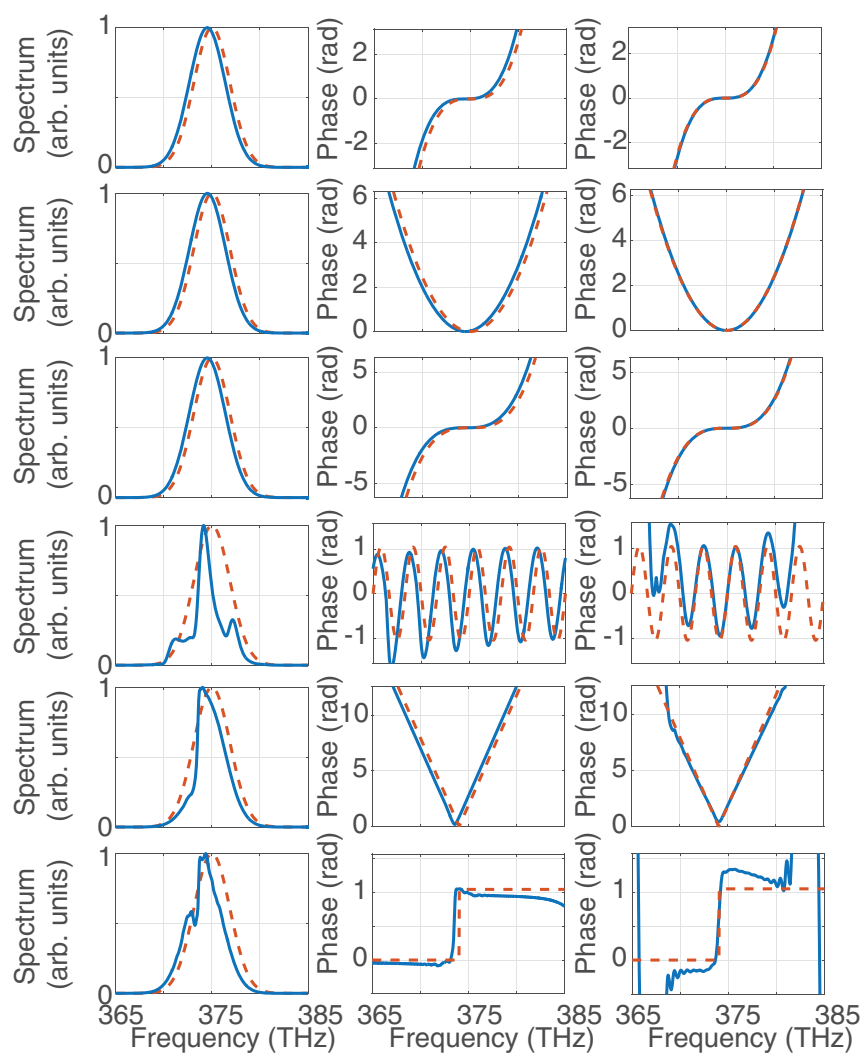

FIG. 8. Simulation of Doppler shift for the different spectral phases experimentally investigated. Left and middle panels depict the spectral amplitude and phase of the input (red dashed line) and sheared pulse (blue solid line). Right panels display the spectral phase obtained from the retrieval procedure (blue solid line) compared to the applied phase (red dashed line).

After some calculations, one then obtains the evolution of the spectral intensity of $I_{+}(\omega)=\left|\widetilde{\varepsilon}_{+}\right|^{2}(\omega)$ and $I_{-}(\omega)=\left|\widetilde{\varepsilon}_{-}\right|^{2}$ :

$$
\begin{aligned}
& \partial_{z} I_{+}(\omega)=\left(\omega_{0}+\omega\right) \frac{n_{2} I_{\mathrm{p}}}{c} \operatorname{Im}\left(\widetilde{\varepsilon}_{-}^{*}\left(\omega+2 \Omega_{\mathrm{rot}}\right) \widetilde{\varepsilon}_{+}(\omega)\right), \\
& \partial_{z} I_{-}(\omega)=-\left(\omega_{0}+\omega\right) \frac{n_{2} I_{\mathrm{p}}}{c} \operatorname{Im}\left(\widetilde{\varepsilon}_{-}^{*}(\omega) \widetilde{\varepsilon}_{+}\left(\omega-2 \Omega_{\mathrm{rot}}\right)\right) .
\end{aligned}
$$

The evolution of the total number of photons is then

$$
\partial_{z} \int \frac{I_{+}(\omega)+I_{-}(\omega)}{\omega_{0}+w} d w=0 .
$$

Accordingly, the total number of photons of the pulse is conserved. Coupled to the fact that there is an energy transfer from the TLP to the characterized pulse (or the opposite), the photon number conservation then implies that the field to characterize is frequency-shifted.

\section{APPENDIX B: THEORETICAL RETRIEVAL}

\section{Phase retrieval}

As already mentioned, the SPIDER consists of measuring the interferogram associated with a sequence of two pulses delayed by $\tau$ and spectrally shifted by $\Omega$ through an interferogram given in Eq. (1). The relevant information is encoded 
in the shape of fringes where the presence of chirp manifests itself as a deviation from equally spaced fringes. The cosine argument can be extracted through a spectral filtering, and the knowledge of $\tau$ allows the evaluation of $\Gamma(\omega)$ that is roughly proportional to the spectral phase derivative. In a first approximation, let us develop $\Gamma(\omega)$ as

$$
\begin{aligned}
\Gamma(\omega) & =\varphi(\omega+\Omega)-\varphi(\omega) \\
& =\left[\varphi(\omega)+\partial_{\omega} \varphi(\omega) \Omega+\frac{1}{2} \partial_{\omega}^{2} \varphi(\omega) \Omega^{2}+\cdots\right]-\varphi(\omega) \\
& =\partial_{\omega} \varphi(\omega) \Omega+\frac{1}{2} \partial_{\omega}^{2} \varphi(\omega) \Omega^{2}+o\left(\Omega^{2}\right) .
\end{aligned}
$$

Integrating $\Gamma$ over $\omega$, one then obtains

$$
\frac{1}{\Omega} \int_{-\infty}^{\omega} \Gamma\left(\omega^{\prime}\right) d \omega^{\prime}=\varphi(\omega)+\frac{1}{2} \partial_{\omega} \varphi(\omega) \Omega+o(\Omega) .
$$

Accordingly, the difference between the pulse phase and the reconstructed phase is proportional to the shear $\Omega$. Moreover, the spectral shape of the systematic error follows the prime derivative of the pulse phase. In fact, the systematic error made during the reconstruction algorithm can be significantly decreased by writing $\Gamma(\omega)$ as

$$
\begin{aligned}
\Gamma(\omega) & =\varphi(\omega+\Omega)-\varphi(\omega) \\
& =\sum_{k=0, \ldots, N} \frac{1}{k !} \partial_{\omega}^{(k)} \varphi\left(\omega+\frac{\Omega}{2}\right) \frac{\Omega^{k}}{2^{k}} \\
& -\sum_{k=0, \ldots, N} \frac{(-1)^{k}}{k !} \partial_{\omega}^{(k)} \varphi\left(\omega+\frac{\Omega}{2}\right) \frac{\Omega^{k}}{2^{k}} \\
& =\partial_{\omega} \varphi\left(\omega+\frac{\Omega}{2}\right) \Omega+\frac{1}{24} \partial_{\omega}^{3} \varphi\left(\omega+\frac{\Omega}{2}\right) \Omega^{3}+o\left(\Omega^{3}\right) .
\end{aligned}
$$

Integrating $\Gamma(\omega)$ over $\omega$, one then obtains

$$
\begin{aligned}
\frac{1}{\Omega} \int_{-\infty}^{\omega} \Gamma\left(\omega^{\prime}\right) d \omega^{\prime}= & \varphi\left(\omega+\frac{\Omega}{2}\right) \\
& +\frac{1}{24} \partial_{\omega}^{2} \varphi\left(\omega+\frac{\Omega}{2}\right) \Omega^{2}+o\left(\Omega^{2}\right) .
\end{aligned}
$$

As a result, considering that the reconstructed phase at a given $\omega$ corresponds to the pulse phase evaluated at $\omega+\frac{\Omega}{2}$, it turns out to be more accurate. Indeed, the error in this case is of second order with respect to the shear frequency $\Omega$ with a frequency shape corresponding to the second-order derivative of the pulse phase. The only additional step for retrieving the correct phase is to shift by $\frac{\Omega}{2}$ the reconstructed phase.

\section{Simulation of phase retrieval}

The above discussion (Appendix A 3) has allowed us to discuss the basic underlying physical processes at play for generating a frequency-shifted replica of the pulse to characterize. Nevertheless, this discussion has been made under the "perfect TLP pulse" assumption, i.e., using a pump pulse whose linear polarization indefinitely rotates at the same angular velocity. However, in practical applications, the TLP pulse is generated from the pulse to characterize. Accordingly, one can legitimately wonder if the above discussion is still valid in the case where the pulse to characterize exhibits a strong, potentially discontinuous, phase modulation. Simulations using propagation equation (Appendix A 4) can provide an answer to this question. The amplitude and phase of the field produced by the Doppler effect for the different shaping explored in this work have been calculated.

Results shown in Fig. 8 reveal that for the quadratic and cubic phases investigated here, the TLP pulses is unaltered so that the Doppler effect provides an exact sheared replica of the input spectrum. For the sinusoidal and triangular phase functions, the alteration of the TLP pulse induces distortions of the spectral amplitude of the sheared pulse while its spectral phase is still well transferred. Since the relevant information is encoded along the fringes spacing and not along the amplitude, the phase retrieval matches the one applied as shown in Figs. 7(b) and 7(g). Finally, simulations for the phase step predict that both the spectral phase and the amplitude of the field produced by the Doppler effect are altered. Nevertheless, the deterioration of the transcribed phase remains moderate, so that the phase retrieval remains satisfactory, as shown in Fig. 7(h).
[1] C. J. Doppler, Ueber das farbige Lichtder Doppelsterne und einiger anderer Gestirne des Himmels [About the coloured light of the binary stars and some other stars of the heavens] (Abhandlungen der Königl. Böhm. Ges. Wiss. V2, 465-482 [Proc. R. Bohemian Soc. Sci. Part V, Vol. 2]) (Prague, 1842, reissued 1903)

[2] F. Durst, B. M. Howe, and G. Richter, Laser-doppler measurement of crosswind velocity, Appl. Opt. 21, 2596 (1982).

[3] R. Lambourne, The doppler effect in astronomy, Phys. Ed. 32, 34 (1997).

[4] Q. Liu, Doppler measurement and compensation in mobile satellite communications systems, in MILCOM 1999, IEEE Military Communications, Conference Proceedings (Cat. No. 99CH36341), 31 Oct.-3 Nov. 1999 (IEEE, Piscataway, NJ, 1999), Vol. 1, pp. 316-320.

[5] S. Agarwal, A. K. Gaurav, M. K. Nirala, and S. Sinha, Potential and sampling based rrt star for real-time dynamic motion planning accounting for momentum in cost function, in Neural Information Processing (Springer International, Cham, 2018), pp. 209-221.

[6] M. Mayor and D. Queloz, A jupiter-mass companion to a solar-type star, Nature (London) 378, 355 (1995).

[7] B. A. Garetz, Angular doppler effect, J. Opt. Soc. Am. 71, 609 (1981).

[8] Q. Miao, O. Travnikova, F. Gel'mukhanov, V. Kimberg, Y.-P. Sun, T. D. Thomas, C. Nicolas, M. Patanen, and C. Miron, Rotational doppler effect: A probe for molecular orbitals anisotropy, J. Phys. Chem. Lett. 6, 1568 (2015).

[9] N. Ashby, Relativity in the global positioning system, Liv. Rev. Relativ. 6, 1 (2003).

[10] O. Korech, U. Steinitz, R. Gordon, I. Sh. Averbukh, and Y. Prior, Observing molecular spinning via the rotational doppler effect, Nat. Photon. 7, 711 (2013). 
[11] G. Karras, M. Ndong, E. Hertz, D. Sugny, F. Billard, B. Lavorel, and O. Faucher, Polarization Shaping for Unidirectional Rotational Motion of Molecules, Phys. Rev. Lett. 114, 103001 (2015).

[12] E. Prost, E. Hertz, F. Billard, B. Lavorel, and O. Faucher, Polarization-based tachometer for measuring spinning rotors, Opt. Express 26, 31839 (2018).

[13] J. Bert, E. Prost, I. Tutunnikov, P. Béjot, E. Hertz, F. Billard, B. Lavorel, U. Steinitz, I. S. Averbukh, and O. Faucher, Optical imaging of coherent molecular rotors, Laser Photon. Rev. 14, 1900344 (2020).

[14] P. Béjot, E. Szmygel, A. Dubrouil, F. Billard, B. Lavorel, Faucher, and E. Hertz, Doppler effect as a tool for ultrashort electric field reconstruction, Opt. Lett. 45, 6795 (2020).

[15] R. Trebino, K. W. DeLong, D. Fittinghoff, J. Sweetser, M. A. Krumbügel, B. Richman, and D. Kane, Measuring ultrashort laser pulses in the time-frequency domain using frequency-resolved optical gating, Rev. Sci. Instrum. 68, 3277 (1997).

[16] I. A. Walmsley and C. Dorrer, Characterization of ultrashort electromagnetic pulses, Adv. Opt. Photon. 1, 308 (2009).

[17] M. Miranda, T. Fordell, C. Arnold, A. L'Huillier, and H. Crespo, Simultaneous compression and characterization of ultrashort laser pulses using chirped mirrors and glass wedges, Opt. Express 20, 688 (2012).

[18] T. Oksenhendler, S. Coudreau, N. Forget, V. Crozatier, S. Grabielle, R. Herzog, O. Gobert, and D. Kaplan, Selfreferenced spectral interferometry, Appl. Phys. B 99, 7 (2010).

[19] M. E. Anderson, A. Monmayrant, S.-P. Gorza, P. Wasylczyk, and I. A. Walmsley, Spider: A decade of measuring ultrashort pulses, Laser Phys. Lett. 5, 259 (2008).

[20] A. Tajalli, T. K. Kalousdian, M. Kretschmar, S. Kleinert, U. Morgner, and T. Nagy, Full characterization of 8 fs deep uv pulses via a dispersion scan, Opt. Lett. 44, 2498 (2019).

[21] T. Nagy and P. Simon, Single-shot tg frog for the characterization of ultrashort duv pulses, Opt. Express 17, 8144 (2009).

[22] S. Linden, J. Kuhl, and H. Giessen, Amplitude and phase characterization of weak blue ultrashort pulses by downconversion, Opt. Lett. 24, 569 (1999).
[23] D. J. Kane, A. J. Taylor, R. Trebino, and K. W. DeLong, Singleshot measurement of the intensity and phase of a femtosecond uv laser pulse with frequency-resolved optical gating, Opt. Lett. 19, 1061 (1994).

[24] P. Londero, M. E. Anderson, C. Radzewicz, C. Iaconis, and I. A. Walmsley, Measuring ultrafast pulses in the near-ultraviolet using spectral phase interferometry for direct electric field reconstruction, J. Mod. Opt. 50, 179 (2003).

[25] P. Baum, S. Lochbrunner, and E. Riedle, Zero-additional-phase spider: Full characterization of visible and sub-20-fs ultraviolet pulses, Opt. Lett. 29, 210 (2004).

[26] A.-C. Tien, S. Kane, J. Squier, B. Kohler, and K. Wilson, Geometrical distortions and correction algorithm in single-shot pulse measurements: application to frequency-resolved optical gating, J. Opt. Soc. Am. B 13, 1160 (1996).

[27] T. C. Wong and R. Trebino, Single-frame measurement of complex laser pulses tens of picoseconds long using pulse-front tilt in cross-correlation frequency-resolved optical gating, J. Opt. Soc. Am. B 30, 2781 (2013).

[28] S. Birkholz, G. Steinmeyer, S. Koke, D. Gerth, S. Bürger, and B. Hofmann, Phase retrieval via regularization in self-diffractionbased spectral interferometry, J. Opt. Soc. Am. B 32, 983 (2015).

[29] B. A. Garetz and S. Arnold, Variable frequency shifting of circularly polarized laser radiation via a rotating half-wave retardation plate, Opt. Commun. 31, 1 (1979).

[30] C. Iaconis and I. A. Walmsley, Spectral phase interferometry for direct electric-field reconstruction of ultrashort optical pulses, Opt. Lett. 23, 792 (1998).

[31] O. Faucher, E. Hertz, B. Lavorel, and F. Billard, Linear and nonlinear optics in coherently spinning molecules, in Progress in Ultrafast Intense Laser Science XIV, Springer Series in Chemical Physics, Vol. 118, edited by K. Yamanouchi, P. Martin, M. Sentis, L. Ruxin, and D. Normand (Springer, Cham, 2018), pp. 37-64.

[32] E. Hertz, F. Billard, G. Karras, P. Béjot, B. Lavorel, and O. Faucher, Shaping of ultraviolet femtosecond laser pulses by fourier domain harmonic generation, Opt. Express 24, 27702 (2016).

[33] C. Dorrer, P. Londero, and I. A. Walmsley, Homodyne detection in spectral phase interferometry for direct electric-field reconstruction, Opt. Lett. 26, 1510 (2001). 